\title{
Adaptive Failure Compensation for Aircraft Tracking Control Using Engine Differential Based Model
}

\author{
Yu Liu, Xidong Tang and Gang Tao \\ Department of Electrical and Computer Engineering \\ University of Virginia \\ Charlottesville, VA 22903
}

\author{
Suresh M. Joshi \\ Mail Stop 308 \\ NASA Langley Research Center \\ Hampton, VA 23681
}

\begin{abstract}
An aircraft model that incorporates independently adjustable engine throttles and ailerons is employed to develop an adaptive control scheme in the presence of actuator failures. This model captures the key features of aircraft flight dynamics when in the engine differential mode. Based on this model an adaptive feedback control scheme for asymptotic state tracking is developed and applied to a transport aircraft model in the presence of two types of failures during operation, rudder failure and aileron failure. Simulation results are presented to demonstrate the adaptive failure compensation scheme.
\end{abstract}

Keywords: Actuator failures, adaptive compensation, aircraft flight control, engine differentials, tracking.

\section{INTRODUCTION}

Effective compensation of control component failures is crucial for aircraft flight safety. Considerable research has focused on the design of control systems that can provide safe performance when failures occur. In [5], an emergency flight control system that can utilize engine thrusts to maneuver an aircraft was developed and tested on an MD11 airplane. In [7], a propulsion controlled aircraft design by $\mathrm{H}$-infinity model matching was introduced. In [1], an indirect adaptive LQ controller was developed for aircraft control, which is able to implicitly reconfigure the control law using on-line estimates of the changed aircraft dynamics, so that the failures in the pitch control channel or the horizontal stabilizer can be accommodated. In [2], several multivariable adaptive control algorithms for flight control reconfiguration were presented with a failure characterized by a locked left horizontal tail surface. An adaptive controller was used to compensate this failure. In [13], a direct adaptive reconfigurable flight control algorithm was presented. An online adaptive neural network was applied to regulate the error between the plant model and the actual aircraft, and application of this control approach to a tailless advanced fighter aircraft was demonstrated. In [9], an algorithm for aircraft failure detection and compensation was presented, which incorporated multiple model adaptive estimation methods. In this approach failures are detected by a bank of parallel Kalman filters and a reconfiguration algorithm is used to redistribute control commands to the non-failed surfaces. In [3], a new parametrization for the modeling of control effector failures in flight control applications was proposed, including lock in place, hard over and loss of effectiveness patterns. An algorithm based on multiple model adaptive reconfiguration control approach was presented and illustrated by simulation results of the F/A-18 aircraft during carrier landing. In [12], an F-16 fighter aircraft subject to asymmetric actuator failure was discussed, including system modeling and control system design. The problem was formulated as a nonlinear disturbance rejection problem in the presence of actuator failures and simulation results using an F-16 aircraft model were discussed. In [6], fault-tolerant control system design against stuck actuators was investigated using an iterative learning observer that provides information of the system state estimates and fault compensation transients. The performance of the controller design was evaluated using an F-8 aircraft model.

In this paper, we present a failure compensation scheme based on an adaptive control approach that can utilize the remaining (functioning) controls to achieve desired performance in the presence of uncertain system failures. To compensate for aircraft failures such as rudder failure or engine malfunction, asymmetric engine thrusts may be inevitably needed [10]. For the design of such control schemes, an aircraft model with independently adjustable engine thrusts is necessary. In [8], we derived such an aircraft model and used it to develop an adaptive failure compensation control scheme using engine differentials for state regulation. In this paper, we shall use this aircraft model to develop an adaptive failure compensation control scheme for state tracking, and apply the scheme to a transport aircraft model. We shall consider two simulation cases representing realistic scenarios in which the rudder or an aileron are stuck at unknown constant values at unknown time instants.

The paper is organized as follows. In Section 2, we describe an engine differential based aircraft flight dynamic model. In Section 3, we develop an adaptive compensation scheme that is able to handle uncertain actuator failures and guarantee asymptotic state tracking. In Section 4, we apply this compensation scheme to a transport aircraft model and present simulation results to illustrate the effectiveness of the scheme.

\section{Engine Differential BASED Model}

As described in [8], a nonlinear aircraft dynamic model in body-axis coordinate system which incorporates engine 
differentials can be described by the force equations

$$
\begin{aligned}
& m(\dot{u}+q w-r v)=X-m g \sin \theta+\left(T_{L}+T_{R}\right) \cos \epsilon \\
& m(\dot{v}+r u-p w)=Y+m g \cos \theta \sin \phi \\
& m(\dot{w}+p v-q u)=Z+m g \cos \theta \cos \phi-\left(T_{L}+T_{R}\right) \sin \epsilon
\end{aligned}
$$

and moment equations

$$
\begin{aligned}
& I_{x} \dot{p}+I_{x z} \dot{r}+\left(I_{z}-I_{y}\right) q r+I_{x z} q p=L+l\left(T_{L}-T_{R}\right) \sin \epsilon \\
& I_{y} \dot{q}+\left(I_{x}-I_{z}\right) p r+I_{x z}\left(r^{2}-p^{2}\right)=M \\
& I_{z} \dot{r}+I_{x z} \dot{p}+\left(I_{y}-I_{x}\right) q p-I_{x z} q r=N+l\left(T_{L}-T_{R}\right) \cos \epsilon
\end{aligned}
$$

where $m$ is the mass of the aircraft. $u, v$ and $w$ are the bodyaxis components of the velocity of the center of mass. $p, q$ and $r$ are the body-axis components of the angular velocity of the aircraft. $X, Y$ and $Z$ are the body-axis aerodynamic forces about the center of mass. $L, M$ and $N$ are the bodyaxis aerodynamic torques about the center of mass. $\theta$ and $\phi$ are the Euler pitch and roll angles of the aircraft. $\epsilon$ represents the angle between thrust and body $x$-axis. $I_{i}$ are the moments (or products) of inertia in body axes. $g$ is the gravitational force per unit mass. $T_{L}$ and $T_{R}$ are the left and right engine thrusts, and $l$ is the distance between engines and $x-z$ plane.

By applying the linearization procedure around the equilibrium point of interest, we can obtain the linearized aircraft model with engine differentials. For this purpose, the state and control vectors of the linearized model are

$$
\begin{aligned}
x & =\left[\begin{array}{lllllllll}
u & w & q & \theta & v & r & p & \phi & \psi
\end{array}\right]^{T} \\
U & =\left[\begin{array}{lllllll}
\delta_{e} & \delta_{t_{l}} & \delta_{t_{r}} & \delta_{a_{l}} & \delta_{a_{r}} & \delta_{r}
\end{array}\right]^{T}
\end{aligned}
$$

where the notation " $\delta$ " has been dropped from $\delta x$ and $\delta U$ for simplicity of presentation. Thus $(u, v, w)$ represent the velocity perturbations along each axis and $(p, q, r)$ are the angular velocity perturbations about each axis. $(\theta, \phi, \psi)$ are the pitch, roll and yaw angle perturbations, and $\delta_{e}, \delta_{a_{l}}, \delta_{a_{r}}$, $\delta_{r}$ are the deflection perturbations of the elevator, the left and right ailerons and the rudder. $\delta_{t_{l}}$ and $\delta_{t_{r}}$ are the left and right throttle perturbations.

In our study, we consider a steady-state rectilinear wingslevel flight condition as the equilibrium point. For this steady-state flight condition, the derivatives of all states, the angular velocity components $(p, q, r)$ and the roll angle $\phi$ at the equilibrium point are all zero, that is,

$$
\begin{gathered}
{\left[\begin{array}{lllllllll}
\dot{u} & \dot{w} & \dot{q} & \dot{\theta} & \dot{v} & \dot{r} & \dot{p} & \dot{\phi} & \dot{\psi}
\end{array}\right]_{x_{o}, U_{o}}=0} \\
p_{o}=q_{o}=r_{o}=\phi_{o}=\psi_{o}=v_{o}=0
\end{gathered}
$$

where $x_{o}$ and $U_{o}$ are determined as

$$
\begin{aligned}
x_{o} & =\left[\begin{array}{lllllllll}
u_{o} & w_{o} & 0 & \theta_{o} & 0 & 0 & 0 & 0 & 0
\end{array}\right]^{T}, \\
U_{o} & =\left[\begin{array}{llllll}
\delta_{e o} & \delta_{t_{l o}} & \delta_{t_{r o}} & \delta_{\text {alo }} & \delta_{\text {aro }} & \delta_{\text {ro }}
\end{array}\right]^{T} .
\end{aligned}
$$

By applying the linearization around this equilibrium point, we can obtain the linearized aircraft model as

$$
\dot{x}=\left[\begin{array}{ll}
A_{4 \times 4}^{(1)} & A_{4 \times 5}^{(2)} \\
A_{5 \times 4}^{(3)} & A_{5 \times 5}^{(4)}
\end{array}\right] x+\left[\begin{array}{cc}
B_{4 \times 3}^{(1)} & B_{4 \times 3}^{(2)} \\
B_{5 \times 3}^{(3)} & B_{5 \times 3}^{(4)}
\end{array}\right] U
$$

where $A^{(2)}$ and $B^{(2)}$ are zero matrices, $A^{(1)}, A^{(4)}, B^{(1)}$ and $B^{(4)}$ are of the same forms as in the literature [4], and the matrices

$$
A^{(3)}=\left[\begin{array}{cccc}
0 & 0 & 0 & 0 \\
\bar{T}_{u} & \bar{T}_{w} & 0 & 0 \\
\bar{T}_{u}^{\prime} & \bar{T}_{w}^{\prime} & 0 & 0 \\
0 & 0 & 0 & 0 \\
0 & 0 & 0 & 0
\end{array}\right], B^{(3)}=\left[\begin{array}{ccc}
0 & 0 & 0 \\
0 & \bar{T}_{t_{t_{l}}}^{\prime \prime} & -\bar{T}_{\delta_{t_{r}}^{\prime \prime}}^{\prime \prime} \\
0 & \bar{T}_{\delta_{t_{l}}}^{\prime \prime \prime} & -\bar{T}_{\delta_{t_{r}}}^{\prime \prime \prime} \\
0 & 0 & 0 \\
0 & 0 & 0
\end{array}\right]
$$

represent the effect of engine thrust differentials, that is, if the left and right engine thrusts are equal, these matrices are zero. See [8] for details of this model.

We note that this aircraft model is different from standard models used in most of the literature [4] that assume equal engine thrusts and aileron angles. This engine differential based model in which the two engine thrusts and the ailerons are taken into account separately captures the essential dynamics of the aircraft in the engine differential mode, and is capable of coping with some actuator failures such as rudder failures or engine failure, which cannot be achieved without using engine differentials. Therefore it is desirable to develop an adaptive control scheme for aircraft actuator failure compensation using engine differentials.

\section{AdAPtive FAILURE COMPENSATION}

In this section, we shall first formulate an actuator failure compensation problem for linear systems, and then develop an adaptive failure compensation scheme for closed-loop stability and asymptotic tracking of the system state variables in the presence of certain actuator failures.

\section{A. Problem Formulation}

Consider the linear time-invariant system

$$
\dot{x}=A x+B u, x \in R^{n}, u \in R^{m},
$$

whose actuators $u=\left[u_{1}, u_{2}, \ldots, u_{m}\right]^{T}$ may fail during system operation. A typical failure model is

$$
u_{i}(t)=\bar{u}_{i}, t \geq t_{i}, i \in\{1,2, \ldots, m\},
$$

where $t_{i}$ is the unknown failure time instant and $\bar{u}_{i}$ is the unknown failure constant [11]. An example of such actuator failures is when an aircraft control surface (such as the rudder or an aileron) is stuck at some unknown fixed position at an unknown time instant.

The control objective is to design an adaptive state feedback control signal to be applied to the actuators in $u$, to ensure closed-loop signal boundedness, and asymptotic tracking: $\lim _{t \rightarrow \infty}\left(x(t)-x_{d}(t)\right)=0$, where $x_{d}(t)$ is a desired state trajectory, in the presence of unknown actuator failures.

\section{B. Adaptive Compensator Designs}

In the presence of actuator failures, $u(t)$ can be expressed as

$$
u(t)=v(t)+\sigma(\bar{u}-v(t))
$$


where $v(t) \in R^{m}$ is the applied control input vector, $\bar{u}=$ $\left[\bar{u}_{1}, \bar{u}_{2}, \ldots, \bar{u}_{m}\right]^{T}$ is the failure vector, and $\sigma$ represents the failure pattern and is defined as

$$
\sigma=\operatorname{diag}\left\{\sigma_{1}, \sigma_{2}, \ldots, \sigma_{m}\right\}
$$

with $\sigma_{i}=1$ if the $i$ th actuator has failed, that is, $u_{i}=\bar{u}_{i}$, and $\sigma_{i}=0$ otherwise. The failures are assumed to occur instantaneously, i.e., $\sigma_{i}$ are piecewise constant functions of time. There are $2^{m}$ possible combinations of actuator states (each actuator is either normal or failed), and therefore $2^{m}-1$ possible failure patterns that constitute a set denoted by $\bar{\Sigma}$. The system (III.1) can then be rewritten as

$$
\dot{x}(t)=A x(t)+B(I-\sigma) v(t)+B \sigma \bar{u} .
$$

For our adaptive control design for actuator failure compensation, the following assumption is needed:

Assumption 3.1: $(A, B)$ is known and stabilizable, and there exists a non-empty set $\Sigma$ of "recoverable" failures such that $\operatorname{rank}[B(I-\sigma)]=\operatorname{rank}[B] \forall \sigma \in \Sigma$. $\Sigma$ is a subset of $\bar{\Sigma}$.

Remark 3.1: This assumption characterizes the built-in redundancy needed for failure compensation as well as all the failure patterns that can be accommodated. This condition is needed for the existence of a (fixed) failure compensation controller that can achieve the desired performance when the system and failure parameters are known. The adaptive control design for unknown failure parameters is developed based on the same condition. For instance, when the aircraft rudder fails during flight, this condition can still be satisfied so that the aircraft can be controlled by the remaining actuators and the failure can be accommodated (which is demonstrated in the simulation in Section 4.2).

For asymptotic tracking, we first present a desired nominal design for the system (III.1) without any actuator failures. The nonadaptive nominal controller is

$$
u(t)=K x(t)+\kappa r_{d}(t),
$$

where $K=-R^{-1} B^{T} P \in R^{m \times n}$ is an optimal LQ gain with $P$ satisfying the Riccati equation

$$
A^{T} P+P A-P B R^{-1} B^{T} P+Q=0
$$

for some chosen $n \times n$ matrix $Q=Q^{T}>0$ and $m \times m$ matrix $R=R^{T}>0$, and the reference input $r_{d}(t) \in R^{m_{r}}$ and $\kappa \in R^{m \times m_{r}}$ are chosen for some desired system trajectory.

With this nominal controller, the closed-loop system is $\dot{x}(t)=(A+B K) x(t)+B \kappa r_{d}(t)$, based on which, we define the desired state trajectory $x_{d}(t)$ from the reference system

$$
\dot{x}_{d}(t)=(A+B K) x_{d}(t)+B \kappa r_{d}(t) .
$$

Define the tracking error $e(t)=x(t)-x_{d}(t)$. As the new adaptive failure compensation scheme for asymptotic state tracking, the feedback control law is

$$
v(t)=\hat{K} x(t)+\hat{\kappa} r_{d}(t)+\hat{\theta},
$$

where $\hat{K}=\left[\hat{K}_{1}, \hat{K}_{2}, \ldots, \hat{K}_{m}\right]^{T} \in R^{m \times n}, \hat{\kappa}=$ $\left[\hat{\kappa}_{1}, \hat{\kappa}_{2}, \ldots, \hat{\kappa}_{m}\right]^{T} \in R^{m \times m_{r}}$, and $\hat{\theta}=\left[\hat{\theta}_{1}, \hat{\theta}_{2}, \ldots, \hat{\theta}_{m}\right]^{T} \in$
$R^{m \times 1}$, are the parameters updated from the adaptive laws

$$
\begin{aligned}
\dot{\hat{K}}_{i} & =-\Gamma_{i} x e^{T} P b_{i}, i=1,2, \ldots, m \\
\dot{\hat{\kappa}}_{i} & =-\gamma_{i} r_{d} e^{T} P b_{i}, i=1,2, \ldots, m \\
\dot{\hat{\theta}}_{i} & =-\lambda_{i} e^{T} P b_{i}, i=1,2, \ldots, m,
\end{aligned}
$$

where $\Gamma_{i}=\Gamma_{i}^{T}>0, \gamma_{i}=\gamma_{i}^{T}>0, \lambda_{i}>0, b_{i}$ is the $i$ th column of $B, i=1,2, \ldots, m$, and $P=P^{T}>0$ satisfying (III.7). $\Gamma_{i}, \gamma_{i}$, and $\lambda_{i}$ denote the design parameters for the adaptive laws. This adaptive actuator failure compensation scheme has the following desired properties:

Theorem 3.1: The control law (III.9), updated from (III.10)-(III.12) and applied to the system (III.1) subject to the actuator failures (III.2) under Assumption 3.1, ensures that all closed-loop system signals are bounded and $\lim _{t \rightarrow \infty}\left(x(t)-x_{d}(t)\right)=0$, for any failure pattern $\sigma \in \Sigma$ with uncertain parameters.

Proof: For $\bar{Q}=Q+P B R^{-1} B^{T} P$, using (III.7), we obtain

$$
P(A+B K)+(A+B K)^{T} P=-\bar{Q}<0 .
$$

Suppose that at time $t$ there are $p<m$ actuator failures, that is, $u_{i}(t)=\bar{u}_{i}, i=i_{1}, i_{2}, \ldots, i_{p},\left\{i_{1}, i_{2}, \ldots, i_{p}\right\} \subset$ $\{1,2, \ldots, m\}$, and that actuator failures happen at time instants $t_{k}$, with $t_{k}<t_{k+1}, k=1,2, \ldots, N$.

From the condition of Assumption 3.1: $\operatorname{rank}[B(I-\sigma)]=$ $\operatorname{rank}[B], \forall \sigma \in \Sigma$, it follows that for each $\sigma \in \Sigma$, there exist constant matrices $K_{\sigma} \in R^{m \times n}$ and $\kappa_{\sigma} \in R^{m \times m_{r}}$ such that

$$
B(I-\sigma) K_{\sigma}=B K, B(I-\sigma) \kappa_{\sigma}=B \kappa .
$$

Therefore, for each $\sigma$, there are constant $K_{\sigma}$ satisfying

$P\left[A+B(I-\sigma) K_{\sigma}\right]+\left[A^{T}+(I-\sigma) K_{\sigma}{ }^{T} B^{T}\right] P=-\bar{Q}<0$, (III.15)

and $\kappa_{\sigma}$ satisfying $B(I-\sigma) \kappa_{\sigma} r_{d}(t)=B \kappa r_{d}(t)$. In addition there exists constant $\theta=\left[\theta_{1}, \theta_{2}, \ldots, \theta_{m}\right]^{T} \in R^{m}$, where $\theta_{i}$, $i \neq i_{1}, i_{2}, \ldots, i_{p}$, are solutions of the following equation

$$
\sum_{i \neq i_{1}, i_{2}, \ldots, i_{p}} b_{i} \theta_{i}=-\sum_{j=i_{1}, i_{2}, \ldots, i_{p}} b_{j} \bar{u}_{j},
$$

and $\theta_{i}=0$, for $i=i_{1}, i_{2}, \ldots, i_{p}$, such that $B(I-\sigma) \theta=$ $B \sigma \bar{u}$.

From (III.5), (III.8), and III.9, we have

$$
\begin{aligned}
\dot{e}(t)= & A x(t)+B(I-\sigma)\left(\hat{K} x(t)+\hat{\kappa} r_{d}(t)+\hat{\theta}\right)+B \sigma \bar{u} \\
& -(A+B K) x_{d}(t)-B \kappa r_{d}(t) \\
= & A x(t)+B(I-\sigma)\left(K_{\sigma} x(t)+\kappa_{\sigma} r_{d}(t)+\theta\right)+B \sigma \bar{u} \\
& -(A+B K) x_{d}(t)-B \kappa r_{d}(t)+B(I-\sigma)\left[\left(\hat{K}-K_{\sigma}\right) x(t)\right. \\
& \left.+\left(\hat{\kappa}-\kappa_{\sigma}\right) r_{d}(t)+(\hat{\theta}-\theta)\right]
\end{aligned}
$$

With equations (III.14) and (III.16), the dynamic equation for tracking error can be simplified as

$$
\begin{aligned}
\dot{e}(t)= & (A+B K) e(t)+B(I-\sigma)\left[\left(\hat{K}-K_{\sigma}\right) x(t)\right. \\
& \left.+\left(\hat{\kappa}-\kappa_{\sigma}\right) r_{d}(t)+(\hat{\theta}-\theta)\right] .
\end{aligned}
$$


With the adaptive laws (III.10)-(III.12), a Lyapunov function candidate can be chosen as

$$
\begin{aligned}
V & =e^{T} P e+\sum_{i \neq i_{1}, i_{2}, \ldots, i_{p}}\left(\hat{K}_{i}-K_{i}\right)^{T} \Gamma_{i}^{-1}\left(\hat{K}_{i}-K_{i}\right) \\
& +\sum_{i \neq i_{1}, i_{2}, \ldots, i_{p}}\left(\hat{\kappa}_{i}-\kappa_{i}\right)^{T} \gamma_{i}^{-1}\left(\hat{\kappa}_{i}-\kappa_{i}\right)+\sum_{\substack{i \neq i_{1}, i_{2}, \ldots, i_{p} \\
i}}^{-1}\left(\hat{\theta}_{i}-\theta_{i}\right)^{2}
\end{aligned}
$$

for each time interval $\left(t_{k}, t_{k+1}\right), k=0,1, \ldots, N$, with $t_{0}=$ 0 and $t_{N+1}=\infty$, where $K_{i}$ is the $i$ th row of $K_{\sigma}$ and $\kappa_{i}$ is the $i$ th row of $\kappa_{\sigma}$. The time-derivative of $V$ in each $\left(t_{k}, t_{k+1}\right)$ is

$$
\begin{aligned}
\dot{V}= & e^{T}\left[P(A+B K)+\left(A^{T}+K^{T} B^{T}\right) P\right] e \\
& +2 e^{T} P B(I-\sigma)\left[\left(\hat{K}-K_{\sigma}\right) x(t)+\left(\hat{\kappa}-\kappa_{\sigma}\right) r_{d}(t)+(\hat{\theta}-\theta)\right] \\
& +2 \sum_{i \neq i_{1}, i_{2}, \ldots, i_{p}}\left(\hat{K}_{i}-K_{i}\right)^{T} \Gamma_{i}^{-1} \hat{\hat{K}}_{i}+2 \sum_{i \neq i_{1}, i_{2}, \ldots, i_{p}}\left(\hat{\kappa}_{i}-\kappa_{i}\right)^{T} \gamma_{i}^{-1} \dot{\hat{\kappa}}_{i} \\
& +2 \sum_{i} \lambda_{i}^{-1}\left(\hat{\theta}_{i}-\theta_{i}\right) \dot{\hat{\theta}}_{i} \\
& i \neq i_{1}, i_{2}, \ldots, i_{p} \\
= & e^{T}\left[P(A+B K)+\left(A^{T}+K^{T} B^{T}\right) P\right] e \\
& +2 e^{T} P B(I-\sigma)\left[\left(\hat{K}-K_{\sigma}\right) x(t)+\left(\hat{\kappa}-\kappa_{\sigma}\right) r_{d}(t)+(\hat{\theta}-\theta)\right] \\
& -2 \sum_{i \neq i_{1}, i_{2}, \ldots, i_{p}}\left(\hat{K}_{i}-K_{i}\right)^{T} x e^{T} P b_{i}-2 \sum_{i \neq i_{1}, i_{2}, \ldots, i_{p}}\left(\hat{\kappa}_{i}-\kappa_{i}\right)^{T} r_{d} e^{T} P b_{i} \\
& -2 \sum_{i \neq i_{1}, i_{2}, \ldots, i_{p}}\left(\hat{\theta}_{i}-\theta_{i}\right) e^{T} P b_{i},
\end{aligned}
$$

For the considered actuator failure pattern, that is, $u_{i}(t)=$ $\bar{u}_{i}, \sigma_{i}=1, i=i_{1}, i_{2}, \ldots, i_{p}$, using the fact that $B(I-$ $\sigma)\left(\hat{K}-K_{\sigma}\right)=\sum_{i \neq i_{1}, i_{2}, \ldots, i_{p}} b_{i}\left(\hat{K}_{i}-K_{i}\right)^{T}$ and the commutativity property of the matrix trace operator, i.e., $\operatorname{Tr}(X Y)=$ $\operatorname{Tr}(Y X)$, the following equalities hold:

$$
\begin{aligned}
e^{T} P B(I-\sigma)\left(\hat{K}-K_{\sigma}\right) x(t) & =\sum_{i \neq i_{1}, i_{2}, \ldots, i_{p}}\left(\hat{K}_{i}-K_{i}\right)^{T} x e^{T} P b_{i}, \\
e^{T} P B(I-\sigma)\left(\hat{\kappa}-\kappa_{\sigma}\right) r_{d}(t) & =\sum_{i \neq i_{1}, i_{2}, \ldots, i_{p}}\left(\hat{\kappa}_{i}-\kappa_{i}\right)^{T} r_{d} e^{T} P b_{i}, \\
e^{T} P B(I-\sigma)(\hat{\theta}-\theta) & =\sum_{i \neq i_{1}, i_{2}, \ldots, i_{p}}\left(\hat{\theta}_{i}-\theta_{i}\right) e^{T} P b_{i} .
\end{aligned}
$$

So the time-derivative of $V$ in each $\left(t_{k}, t_{k+1}\right)$ is simplified as

$$
\dot{V}=-e^{T} \bar{Q} e \leq 0,
$$

where (III.13) is used for the last equality. It follows that $e \in$ $L^{2} \cap L^{\infty}$, and $\hat{K}_{i} \in L^{\infty}$ and $\hat{\theta}_{i} \in L^{\infty}$ for $i \neq i_{1}, i_{2}, \ldots, i_{p}$, where $i_{1}, i_{2}, \ldots, i_{p}$ are the indexes of failed actuators.

From (III.10)-(III.12), we have

$$
\begin{array}{r}
{\left[\Gamma_{1}^{-1} \dot{\hat{K}}_{1}, \Gamma_{2}^{-1} \dot{\hat{K}}_{2}, \ldots, \Gamma_{m}^{-1} \dot{\hat{K}}_{m}\right]=-x e^{T} P B} \\
{\left[\gamma_{1}^{-1} \dot{\hat{\kappa}}_{1}, \gamma_{2}^{-1} \dot{\hat{\kappa}}_{2}, \ldots, \gamma_{m}^{-1} \dot{\hat{\kappa}}_{m}\right]=-r_{d} e^{T} P B} \\
{\left[\lambda_{1}^{-1} \dot{\hat{\theta}}_{1}, \lambda_{2}^{-1} \dot{\hat{\theta}}_{2}, \ldots, \lambda_{m}^{-1} \dot{\hat{\theta}}_{m}\right]=-e^{T} P B}
\end{array}
$$

which implies that $\hat{K}_{i} \in L^{\infty}, \hat{\kappa}_{i} \in L^{\infty}$ and $\hat{\theta}_{i} \in L^{\infty}$ for $i=i_{1}, i_{2}, \ldots, i_{p}$, because $B$ can be represented by a linear combination of $b_{i}, i \neq i_{1}, i_{2}, \ldots, i_{p}$.

The function $V$ is not continuous at $t_{k}, k=0,1, \ldots, N$, and only has finite value jumps at those time instants. So we can conclude that $e \in L^{2} \cap L^{\infty}, \hat{K} \in L^{\infty}, \hat{\kappa} \in L^{\infty}$, and $\hat{\theta} \in L^{\infty}$. For the nominal design (III.6), $A+B K$ is asymptotically stable such that $x_{d}(t) \in L^{\infty}$ with a bounded reference input $r_{d}(t)$. Hence we conclude that $x(t) \in L^{\infty}$, so does $v(t)$. Furthermore, since $\dot{x}(t) \in L^{\infty}$ and $\dot{x}_{d} \in L^{\infty}$, given that $e(t) \in L^{2}$, we also have $\lim _{t \rightarrow \infty} e(t)=0$.

The physical meaning of state tracking for the aircraft dynamic model linearized at an equilibrium point $\left(x_{o}, U_{o}\right)$ is that the operation of the aircraft follows a desired trajectory in a neighborhood of the equilibrium point. When we apply this adaptive failure compensation scheme to aircraft flight control, we want the aircraft to maintain the desired trajectory that was originally set for the nominal case of no failure, even if unknown actuator failures occur. Theorem 3.1 gives a solution to the problem of state tracking. The stabilizability and rank condition in Assumption 3.1 characterizes the system redundancy condition needed for actuator failure compensation. As shown in next section, it is satisfied for the rudder or aileron failure case and the system state is able to track the desired trajectory asymptotically, which implies that the aircraft can maintain the desired performance under normal as well as failure conditions. Assumption 3.1 also requires the knowledge of $A$ and $B$. However, it may be noted that, if the actual system matrix given by $A_{p}=A+\delta A$ (where $\delta A$ is the parameter error) is such that $\left(A_{p}+B K\right)^{T} P+P\left(A_{p}+B K\right)<0$, the asymptotic tracking and signal boundedness of Theorem 3.1 will still hold. (This condition basically implies that the nominal LQ regulator used for generating the desired trajectory is designed to be robust to parameter uncertainties). Further research is needed in order to investigate robustness of the adaptive scheme to model errors, and to relax the requirement of knowledge of $A$ and $B$.

\section{Application to Flight Control}

In this section, we demonstrate application of the adaptive failure compensation technique to a transport aircraft by presenting some simulation results for trajectory tracking in the presence of unknown rudder and aileron failures. We shall first describe the aircraft model used in simulation, and then present the simulation results.

\section{A. Aircraft Model for Simulation Study}

For our simulation study, we use a transport aircraft model. The airplane flies at a velocity of $774 \mathrm{ft} / \mathrm{sec}$ and an altitude of $40 \mathrm{kft}$. The linearized dynamic model is

$$
\dot{x}(t)=\left[\begin{array}{ll}
A_{4 \times 4}^{(1)} & A_{4 \times 5}^{(2)} \\
A_{5 \times 4}^{(3)} & A_{5 \times 5}^{(4)}
\end{array}\right] x(t)+\left[\begin{array}{cc}
B_{4 \times 3}^{(1)} & B_{4 \times 3}^{(2)} \\
B_{5 \times 3}^{(3)} & B_{5 \times 3}^{(4)}
\end{array}\right] U(t)(\mathrm{IV} .1)
$$

where $A^{(2)}$ and $B^{(2)}$ are zero matrices, and $A^{(1)}, A^{(4)}, B^{(1)}$ and $B^{(4)}$ are of the same forms as in [8], and

$$
\begin{aligned}
& x=\left[\begin{array}{lllllllll}
u & w & q & \theta & v & r & p & \phi & \psi
\end{array}\right]^{T}, \\
& U=\left[\begin{array}{llllll}
\delta_{e} & \delta_{t_{l}} & \delta_{t_{r}} & \delta_{a_{l}} & \delta_{a_{r}} & \delta_{r}
\end{array}\right]^{T}, \\
& A^{(3)}=\left[\begin{array}{cccc}
0 & 0 & 0 & 0 \\
0.001 & 0.001 & 0 & 0 \\
-0.001 & -0.001 & 0 & 0 \\
0 & 0 & 0 & 0 \\
0 & 0 & 0 & 0
\end{array}\right], B^{(3)}=\left[\begin{array}{ccc}
0 & 0 & 0 \\
0 & 0.8 & -0.7 \\
0 & -0.5 & 0.6 \\
0 & 0 & 0 \\
0 & 0 & 0
\end{array}\right]
\end{aligned}
$$


The non-zero terms in $A^{(3)}$ and $B^{(3)}$ represent the engine thrust differential effect. The basic units used in this model are $\mathrm{ft}$, sec and crad (0.01 radian).

We consider two types of constant actuator failures: rudder failure and aileron failure. The rudder failure is denoted as

$$
U_{6}(t)=U_{6}\left(t_{f}\right) \quad t \geq t_{f},
$$

where $t_{f}$ is the failure time instant. It represents the rudder stuck in its position at instant $t_{f}$, and cannot be moved. The aileron failure we consider is

$$
U_{5}(t)=0 \quad t \geq t_{f},
$$

which indicates that at failure time instant $t_{f}$, the right aileron angle drops to zero and is stuck from then on. These failure patterns satisfy Assumption 3.1.

\section{B. Simulation Results}

In this subsection, we present the simulation results for the asymptotic tracking of $x_{d}(t)$ by $x(t)$ to demonstrate the performance of the system with the adaptive failure compensation scheme described in Section 3.2. For our simulation, the values of $\kappa$ and $r_{d}$ were chosen as $r_{d}=1$, and

$\kappa=\left[\begin{array}{llllll}0.7344 & 0.6842 & 1.5974 & -0.5817 & -0.7853 & 1\end{array}\right]^{T}$

that is, $r_{d}=r_{d}^{0} \in R$ is a scalar, which leads to the final values of the desired states:

$$
x_{d}^{\infty}=\left[\begin{array}{lllllllll}
4 & -1.06 & 0 & 1 & 0 & 0 & 0 & 0 & -1.8
\end{array}\right]^{T}
$$

This trajectory represents a steady state flight condition in which the aircraft is climbing with a $4 \mathrm{ft} / \mathrm{sec}$ velocity perturbation along the $x$-axis, a $-1.06 \mathrm{ft} / \mathrm{sec}$ velocity perturbation along the $z$-axis, a pitch angle of 1 crads $(0.57$ degrees), and a yaw angle of -1.8 crads (-1.0 degree). The initial value of the state vector is zero, i.e., the airplane is in steady wings-level flight. The physical meaning of state tracking is that the aircraft flies from one steady state flight condition to another steady state flight condition while closely following a reference state-trajectory. The choice of the reference trajectory (in particular, $\kappa$ and $r_{d}$ ) in this paper was arbitrary, the main purpose being demonstration of the adaptive scheme. For the controller design, we choose $Q=I_{9}$ and $R=\operatorname{diag}\{2,6,6,2,2,2\}$.

For our simulation study, we examine two cases: (I) system responses with adaptive failure compensation with failure (IV.2) and (II) system responses with adaptive failure compensation with aileron failure (IV.3).

Case (I). System performances with adaptive compensation scheme and rudder failure (IV.2). The failure instant is $t_{f}=5$ seconds. $\Gamma_{i}(i=1, \ldots, 6)$ are chosen as $\left[\begin{array}{lllllllll}0.01 & 0.01 & 0.01 & 0.06 & 0.01 & 0.01 & 0.01 & 0.04 & 0.08\end{array}\right]$. $\gamma_{i}$ and $\lambda_{i}(i=1, \ldots, 6)$ are chosen as $\left[\begin{array}{llllll}0.01 & 0.05 & 0.05 & 0.02 & 0.02 & 0.01\end{array}\right]$. These design parameters were chosen by trial and error. Some selected states and control signals are shown in Figures 1 and 2, which demonstrate how the rudder failure is accommodated
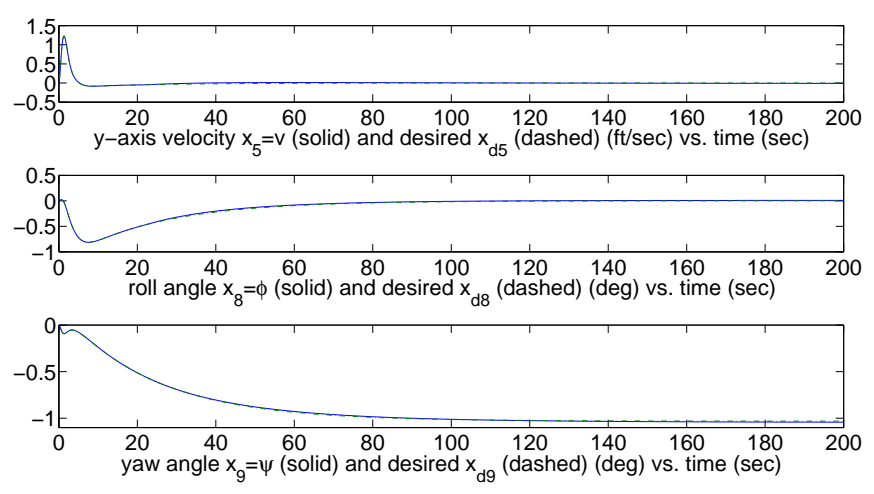

Fig. 1. System states $x_{5}=v, x_{8}=\phi, x_{9}=\psi($ Case I).
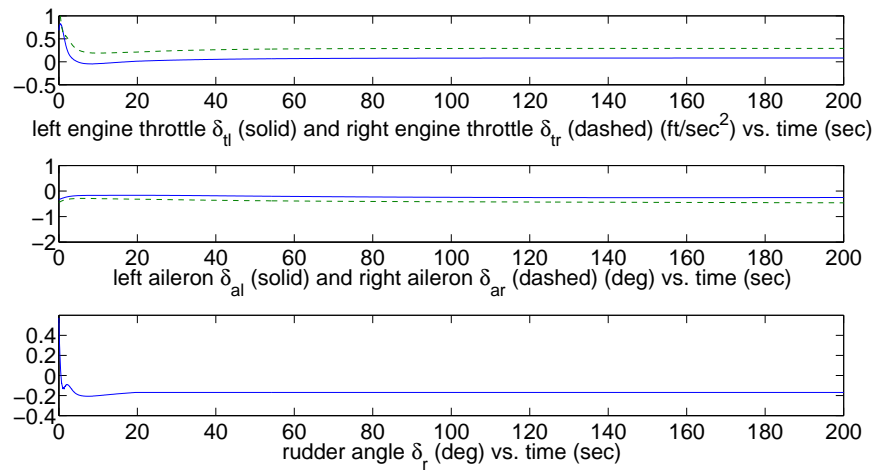

Fig. 2. Control signals: $\delta_{t l}, \delta_{t r}, \delta_{a l}, \delta_{a r}$, and $\delta_{r}$ (Case I).

immediately after its occurrence by the adaptive controller. (The other states can also converge to the desired trajectory after rudder failure, but are not shown in the figures due to the limitation of space). The initial transients (before the failure occurs) are because of the adaptive system response when the system is first turned on with some arbitrary initial values of the adaptive control gains.

Case (II). System performances with adaptive compensation scheme and aileron failure (IV.3). The failure instant is $t_{f}=20$ seconds and the parameter setting is the same as Case (I). The results are shown in Figures 3 and 4.
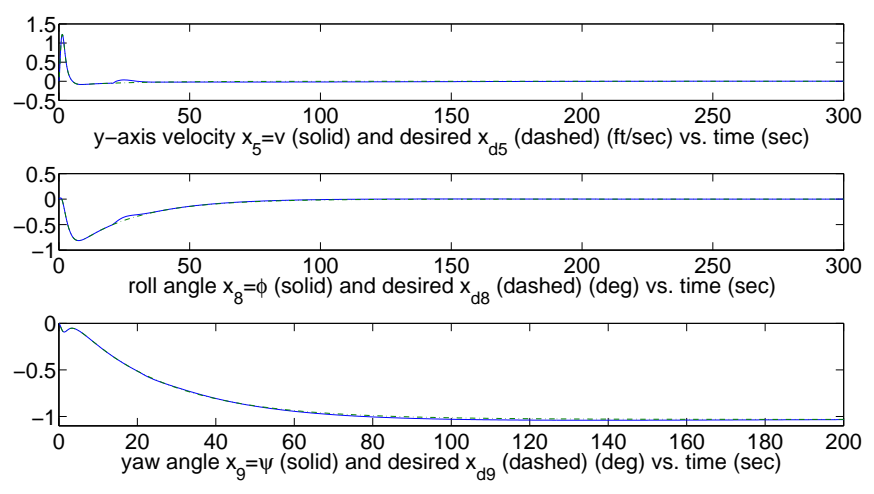

Fig. 3. System states $x_{5}=v, x_{8}=\phi, x_{9}=\psi$ (Case II).

In summary, in this study we simulated some typical aircraft motions for realistic rudder and aileron failure con- 


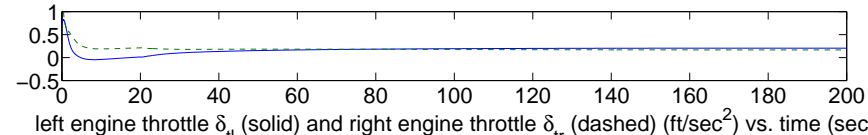

left engine throttle $\delta_{\mathrm{tl}}$ (solid) and right engine throttle $\delta_{\mathrm{tr}}$ (dashed) $\left(\mathrm{ft} / \mathrm{sec}^{2}\right.$ ) vs. time (sec)
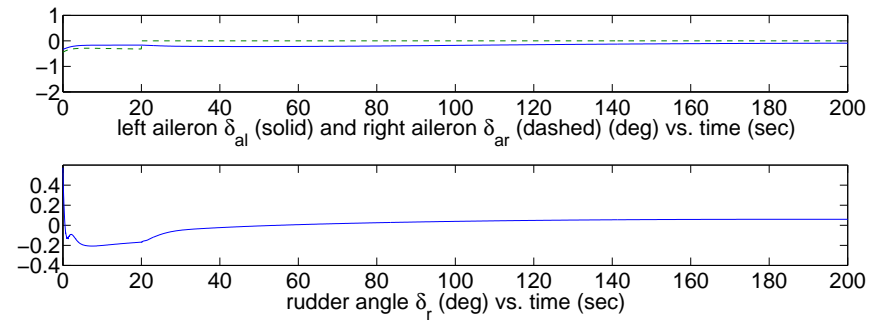

Fig. 4. Control signals: $\delta_{t l}, \delta_{t r}, \delta_{a l}, \delta_{a r}$, and $\delta_{r}$ (Case II).

ditions. The failure uncertainties are characterized by the failure value and failure time instant: both are unknown to the adaptive controller. Simulation results indicate the ability of the adaptive compensation scheme to accommodate unknown actuator failures and to maintain the desired performance regardless of whether a failure has occurred or not, and the value of the failure. This objective cannot be achieved with a fixed controller. We can see from the simulation results that the engine differentials and ailerons, which characterize the system redundancy mentioned previously, make the main contribution to the failure compensation so as to achieve the control objectives. The same goal cannot be achieved without using engine differentials.

\section{CONCLUding REMARKS}

A dynamic model of aircraft with independently adjustable engine throttles and ailerons was considered for failure compensation in the presence of rudder or aileron failure. This model captures the key features of aircraft flight dynamics when in the engine differential mode and facilitates the development of an adaptive failure compensation approach to handle actuator failures using functioning actuators that can be of types different from the failed actuators. An adaptive actuator failure compensation scheme was proposed, which guarantees closed-loop signal boundedness as well as asymptotic state tracking in the presence of unknown actuator failures occurring at unknown time instants. Simulation results obtained for a large transport aircraft model indicate that the adaptive scheme can provide satisfactory performance in the presence of rudder or aileron failures, i.e., the functioning actuators automatically and seamlessly take over for the failed ones.

Several important and challenging issues need to be addressed in future research. First, robustness of the adaptive scheme to model errors, and relaxation of the requirement of knowledge of the system matrices, need to be investigated. In addition, the effects of actuator nonlinearities including output and rate saturation, as well as actuator dynamics, need to be addressed. Also, extensions of the adaptive scheme to model reference state and output tracking, as well as to nonlinear aircraft models, should be addressed.

\section{ACKNOWLEDGMENTS}

This research was partially supported by NASA Langley Research Center under grant NCC-1-02006. The authors would like to thank the reviewers for their helpful comments.

\section{REFERENCES}

[1] Ahmed-Zaid, F., P. Ioannou, K. Gousman, and R. Rooney, "Accommodation of failures in the F-16 aircraft using adaptive control," IEEE Control Systems Magazine, vol. 11, no. 1, pp. 73-78, 1991.

[2] Bodson, M. and J. E. Groszkiewicz, "Multivariable adaptive algorithms for reconfigurable flight control," IEEE Transactions on Control Systems Technology, vol. 5, no. 2, pp. 217-229, March, 1997.

[3] Bošković, J. D., R. K. Mehra, "Multiple-model adaptive flight control scheme for accommodation of actuator failures," Journal of Guidance, Control and Dynamics, vol. 25, no. 4, 2002.

[4] Bryson, A. E., Jr., Control of Spacecraft and Aircraft, Princeton University Press, Princeton, NJ, 1994.

[5] Burcham, F. W., J. J. Burken, T. A. Maine and C. G. Fullerton, "Development and Flight Test of an Emergency Flight Control System Using Only Engine Thrust on an MD-11 Transport Airplane," NASA/TP-97-206217, Dryden Flight Research Center, 1997.

[6] Chen, W and J. Jiang, "Fault-tolerant control against stuck actuator faults," IEE Proc. of Control Theory Applications, vol. 152, no. 2, pp. 138-146, March 2005.

[7] Jonckheere, E. A. and G. R. Yu, "Propulsion control of crippled aircraft by $H_{\infty}$ model matching," IEEE Transactions on Control Systems Technology, vol. 7, no. 2, pp. 142-159, March, 1999.

[8] Liu, Y., X. Tang, G. Tao and S. M. Joshi, "Adaptive Rudder Failure Compensation for Aircraft Flight Control Using Engine Differentials: Regulation,”Proc. of Infotech@Aerospace Conference, Arlington, VA, 2005.

[9] Maybeck, P. S., "Multiple model adaptive algorithms for detecting and compensation sensor and actuator/surface failures in aircraft flight control systems," Int. J. Robust Nonlinear Control, no.9, pp. 1051-1070, 1999.

[10] National Transportation Safety Board, "Aircraft Accident Report: United Airlines Flight 232, McDonnell Douglas DC-10-10, Sioux Gateway Airport, Sioux City, Iowa, July 19, 1989," National Transportation Safety Board, NTSB/AAR-90/06.

[11] Tao, G., S. Chen, X. Tang and S. M. Joshi, Adaptive Control of Systems with Actuator Failures, Springer-Verlag, London, 2004.

[12] Thomas, S., H. G. Kwatny and B. C. Chang, "Nonlinear reconfiguration for asymmetric failures in a six degree-of-freedom F-16," Proc. of the 2004 American Control Conference, pp. 1823-1828.

[13] Wise, K., J. S. Brinker, A. J. Calise, D. F. Enns and M. R. Elgersma, "Direct adaptive reconfigurable flight control for a tailless advanced fighter aircraft," Int. J. Robust and Nonlinear Control, vol. 9, pp. 999-1009, 1999. 